\section{Spondyloarthritis: may the force be with you?}

\author{
Dennis McGonagle, ${ }^{1,2}$ Rebecca C Thomas, ${ }^{1}$ Georg Schett ${ }^{3}$
}

In this issue Jacques et al ${ }^{1}$ provide proof of concept that experimental spondyloarthritis (SpA) may be a biomechanically triggered process. The implication is that an aberrant mesenchymal tissue response to physiological mechanical forces that occur during joint movement may trigger inflammation associated with SpA. Given the long-known genetic association of human SpA with human leukocyte antigen (HLA)-B27, ${ }^{2}$ which governs initiation of adaptive immune responses, the concept that $\mathrm{SpA}$ resembles a biomechanically driven rather than autoimmune disease may come as a great surprise. The en face argument that mechanically driven animal models bear no resemblance to human $\mathrm{SpA}$, however, has to be taken with caution, given that the role of mechanics in human SpA has several supporting strands of evidence that date back over 50 years.

Jacques and colleagues show that the earliest lesions in their model are localised at the Achilles tendon. As far back as 1959, La Cava, in his prophetic commentary on enthesitis, but not specifically in relationship to $\mathrm{SpA}$, recognised that "the continually recurring concentration of muscle stress at these points provokes a reaction of inflammation with a strong tendency to the formation of fibrosis and calcification". ${ }^{3} \mathrm{He}$ also stated that insertions appeared to show 'a peculiar reaction to irritative stimuli' and that such stimuli were most frequently 'microtraumatic in origin'. Since that time evidence has accumulated that supports all aspects of these suppositions.

In the 1970s, Moll and Wright deduced the clinical concept of SpA. ${ }^{4-6}$ Although they did not pinpoint the 'invisible unifying concept', which appears to be mechanical stress, they recognised that physical trauma to joints appeared to trigger psoriatic arthritis and speculated that psoriatic arthritis

\footnotetext{
${ }^{1}$ Leeds Institute of Rheumatic and Musculoskeletal Medicine, University of Leeds, Leeds, UK; ${ }^{2}$ Leeds Musculoskeletal and Biomedical Research Unit, Leeds Teaching Hospitals NHS Trust, Chapel Allerton Hospital, Leeds, UK; ${ }^{3}$ Department of Medicine III, University Hospital of Erlangen, Erlangen, Germany
}

Correspondence to Professor D G McGonagle, Leeds Musculoskeletal and Biomedical Research Unit, Leeds Teaching Hospitals NHS Trust, Chapel Allerton Hospital, Leeds LS7 4SA, UK; d.g.mcgonagle@leeds.ac.uk
(PsA) represented a 'Deep Koebner response'. However, they did not specifically recognise the importance of the entheseal organ as the link between biomechanics and inflammation. Likewise, nail disease in psoriasis shows a propensity for the thumbs and index fingers compared with adjacent nails, ${ }^{7}$ pointing to the importance of mechanical factors and resident tissue responses in the initiation and clinical gestalt of disease. The differing clinical patterns of $\mathrm{SpA}$ with the propensity for large joints in the lower limbs in adult disease and the propensity for foot involvement in juvenile SpA both attest to factors linked to resident cells and tissue as being the key determinants of the disease process of SpA.

In 1984, Bywaters suggested that morbid pathology had little left to offer in the study of SpA, which to him appeared as a mechanically driven disease given the propensity for disease localisation to the enthesis and to the aortic root. ${ }^{8}$ Noting that clinically occult enthesitis was common in inflamed synovial joints in $\mathrm{SpA}$ - and noting that perientheseal osteitis was common in SpA and that its distribution often equated with apparent bone stressing patterns-we proposed that human SpA may be primarily a biomechanically driven disease with secondary adaptive immune response modifying the clinical phenotype. ${ }^{9}$

The entheseal organ is the visible face of complex joint mechanical stressing, but there are other important locations that exhibit similar features. These include wrap around tendons and fibrocartilagenous synovial joints, both of which also show a proclivity for disease localisation in man and in experimental models of arthritis. ${ }^{10}$ In the present study, Jacques et al used a well-established tumour necrosis factor (TNF) transgenic model that develops a Crohn's-like colitis and arthritis (both sacroiliitis and peripheral arthritis) to show that disease localises primarily to the enthesis. They also provided MRI evidence of sacroiliac joint osteitis, although this finding was not histologically confirmed.

The actual joint unloading was performed using a tail suspension procedure with the animals being placed at $30^{\circ}$, which apparently permitted some limited contact with the ground. Careful histological evaluation in early disease confirmed an enthesitis-based peripheral arthritis at three different locations, including interphalangeal joints, Achilles tendons and the hip joints. As previously shown by this group, arthritis development was independent of the adaptive immune response as it also developed in RAG1 knockout mice, which lack $\mathrm{T}$ lymphocytes. ${ }^{11}$ Histological evaluation of animals in the later stages of disease showed extensive secondary synovitis and bone erosion. Remarkably the joint unloading procedure almost completely blocked the arthritis development, and furthermore no increase of arthritis was reported in the anterior limbs, which were not unloaded. The TNF transgenic mouse model is not characterised by significant new bone formation, ${ }^{12}$ and consequently the authors explored the effect of tail suspension in the SpA model of male DBA1 mice. Again, tail suspension alleviated the course of arthritis that was associated with marked reduction of new bone formation at the enthesis. Therefore, mechanical stress appears to play a significant role in both inflammation and new bone formation in different model systems.

In order to explore the molecular basis for these findings, the authors investigated those intracellular signalling pathways that have previously been shown to be responsive to mechanical stress in stromal and other cell types. ${ }^{13-16}$ Specifically, ERK1 and ERK2, two mitogen-activated protein kinases, appear to be important signalling components linking biomechanical stress to the development of enthesitis. However, given the multiplicity of functions of ERKs, including mediating the cytokine signalling, it remains unclear whether ERK inhibition directly antagonises the mechanical stress response or indirectly through modulation of the inflammatory response.

The findings of Jacques and colleagues revitalise the concept of biomechanical factors translating to biochemical tissue responses and envision a pivotal role of mechanical stress in the pathogenesis of human SpA. Several questions arise from these findings:

1. How do genetic factors, particularly the HLA-B27 and the IL-23 pathway, influence this process? ${ }^{2} 1718$ It can be speculated that genetic factors in $\mathrm{SpA}$ patients lower the threshold of pathological mechanical stress, which renders entheseal organs more vulnerable to physical forces. Alternatively, genetic factors may solely alter the inflammatory response to stress but not tissue 


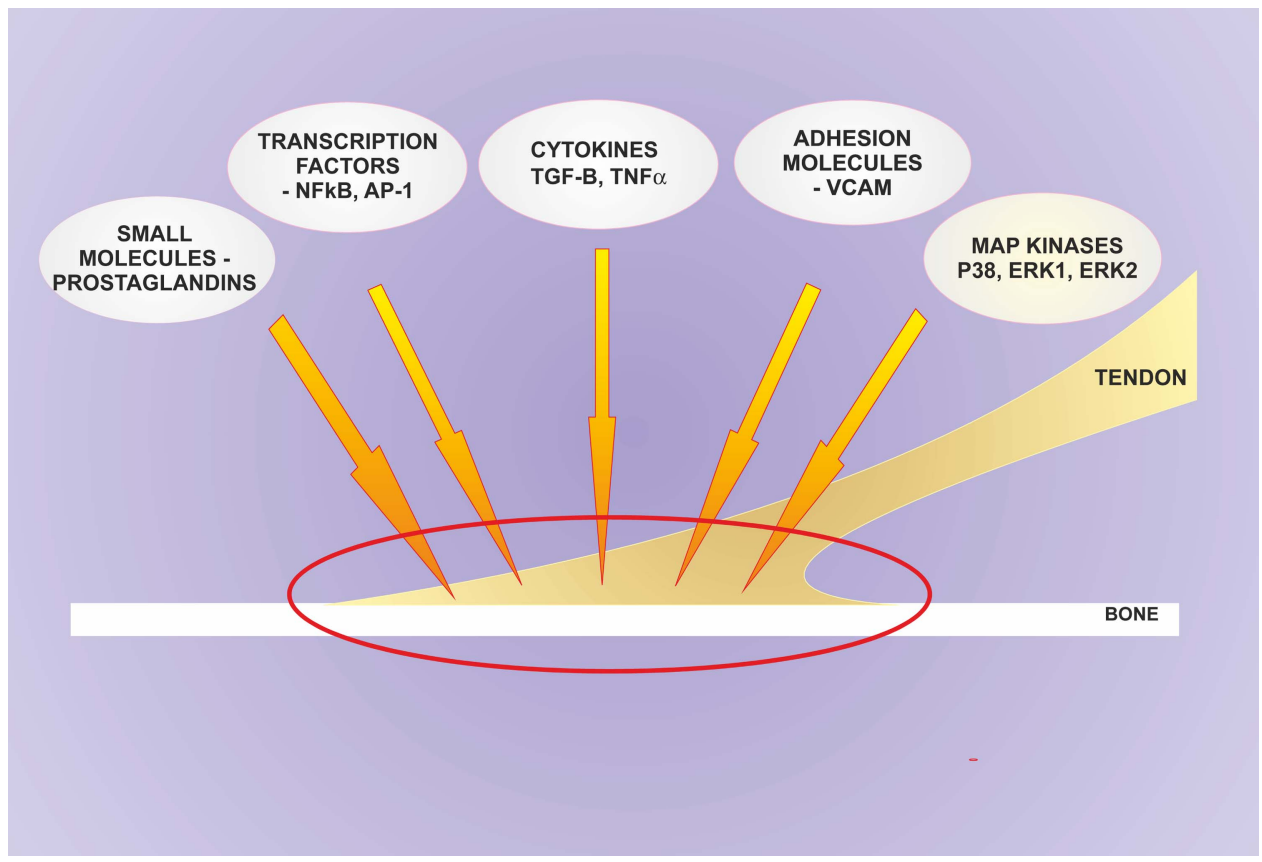

Figure 1 The response to mechanical stress at the enthesis is not merely confined to the point of attachment but also involves the immediately adjacent tissues of the enthesis organ, including the bone, extra-enthesis fascia, synovio-entheseal complex and bone (all regions inside the red circle). It turns out that many metabolic/cytokine pathways are shared by the immune system and also regulated by mechanical stress. This is well recognised in cardiovascular medicine but increasingly so in the rheumatic diseases. These pathways include transcriptional factors, mitogen-activated protein kinases and other kinase pathways, cytokines including tumour necrosis factor- $\alpha^{26}$ and small molecules including prostaglandins. AP-1, activator protein 1; TGF-B, transforming growth factor beta; VCAM, vascular cell adhesion molecule 1.

susceptibility to stress. Sites of complex patterns of biomechanical stress, including entheses and synovial joints such as the SIJ, are associated with induction of fibrocartilage rather than articular cartilage. In human disease, there is a link between carriage of the HLA-B27 gene and osteitis adjacent to fibrocartilage. ${ }^{19}$ Although the present animal model is independent of adaptive immunity, in man it has been suggested that autoimmunity against fibrocartilage at these sites of stress could be an initiating event. ${ }^{20}$

2. Does mechanical stress per se drive disease or are microdamage and injury consequent to such stress the primary triggers for inflammation? Bone responses and the associated bone modelling pathways such as Wnt proteins and their antagonists, the Hedgehog pathway and bone morphogenic proteins may represent important mechanistic links between biomechanical stress and inflammation. ${ }^{12} 21$ Classical autoimmune diseases such as rheumatoid arthritis (RA) may also ameliorate following joint rest or hemiplegic strokes. $^{22}{ }^{23}$ However, RA is typically preceded by autoantibody production for many years, which likely points towards mechanical stress as an aggravating factor rather than as an initiator.

3. Do we have to reconsider the role of exercise in SpA? Exercise presently forms the cornerstone of comprehensive management of SpA patients in conjunction with anti-inflammatory drug therapy. ${ }^{24}$ Clinical experience suggests that exercise and associated mechanical strain may reduce inflammation in SpA fostering efficacy of anti-inflammatory drug treatment. Considering the data from Jacques and colleagues, exercise may in fact be a double-edged sword in patients with SpA. It thus remains to be determined which kind of mechanical loading is beneficial for SpA and which one is not.

4. How might a disease with a primary mechanical stress mediated initiation subsequently unfold in human $\mathrm{SpA}$ that for many facets of disease is linked to HLA B-27 and to abnormal mucosal permeability, especially of the gut? First, abnormal gut permeability is typically associated with access of bacterial adjuvant to the circulation. It has been suggested that the proinflammatory signals associated with aberrant responses to mechanical stress in addition to those derived from adjuvant from the gut or other sights might lead to innate immune activation at the enthesis and other sites of high stressing. 9 The migration of primed dendritic cells to regional nodes and the presentation of arthritogenic peptides via HLA-B27 could then lead to secondary adaptive immune response and multilevel spinal inflammation in the case of ankylosing spondylitis (AS) and axial HLA-B27-associated SpA. However, as shown in Jacques et al's paper, the SpA-like phenotype can occur with equal severity in the absence of an adaptive immune system. So the role of mechanical stress as a dominant factor needs much scrutiny in non-HLA-associated $\mathrm{SpA}$ to determine whether such disease entities may be predominantly exclusively mediated by innate immune mechanisms.

5. From the medicolegal perspective, an association between frank joint injury with associated structural damage and the subsequent development of entities such as osteoarthritis and PsA has been accepted. Now these findings raise the possibility that the magnitude of injury, if indeed it can be demonstrated at all, may be rather small. The threshold for injury in the 'medicolegal immunology field' of inflammatory arthritis would appear to have been considerably lowered.

The present study raises intriguing yet unexplained and unresearched possibilities with respect to human SpA. Why is disease not more common in obese individuals possessing HLA-B27? Why is pregnancy not an exacerbating factor for axial inflammation? Why does inflammation appear in apparently totally ankylosed segments of the spine on MRI if biomechanical factors are relevant to susceptibility 
and severity of disease? Most of these questions have not been addressed, but it is interesting to note that one study showed a much worse disease course in AS in subjects who had occupationally related heavy lifting activities. ${ }^{25}$ The present study will hopefully spur further investigation of these questions.

In summary, the work by Jacques and colleagues has rediscovered the importance of mechanical components of musculoskeletal disease showing us how tightly such factors are related to inflammation. The data suggest that in order to understand the pathogenesis of SpA one needs to better address the liaison between these mechanical factors and the inflammatory cytokine network (figure 1). In a disease process, where the pathology primarily affects the organ of mechanotransduction from muscle to bone, physical force cannot be neglected as a component of the disease process. To understand this force and make it useful for the SpA patients will be the key area of future research in the SpA field.

Competing interests None.

Provenance and peer review Commissioned; externally peer reviewed.

To cite McGonagle D, Thomas RC, Schett G. Ann Rheum Dis 2014;73:321-323.

Received 2 July 2013

Revised 23 October 2013

Accepted 24 October 2013

Published Online First 12 November 2013

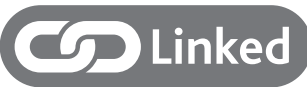

http://dx.doi.org/10.1136/annrheumdis-2013203643
Ann Rheum Dis 2014:73:321-323.

doi:10.1136/annrheumdis-2013-203924

\section{REFERENCES}

1 Jacques $P$, Lambrecht $S$, Verheugen $E$, et al. Proof of concept: enthesitis and new bone formation in spondyloarthritis are driven by mechanical strain and stromal cells. Ann Rheum Dis 2014;73:437-45.

2 Schlosstein L, Terasaki PI, Bluestone R, et al. High association of an $\mathrm{HL}-\mathrm{A}$ antigen, W27, with ankylosing spondylitis. N Engl J Med 1973;288:704-6.

3 La Cava G. Enthesitis; traumatic disease of insertions. J Am Med Assoc 1959;169:254-5.

4 Wright V. Psoriatic arthritis. In: Scott J, ed. Copeman's textbook of the rheumatic diseases. 5th edn. Edinburgh: Churchill Livingstone, 1978:537-48.

5 Moll JM, Wright V. Familial occurrence of psoriatic arthritis. Ann Rheum Dis 1973;32:181-201.

6 Moll JM, Wright V. Psoriatic arthritis. Semin Arthritis Rheum 1973:3:55-78.

7 Rich P, Griffiths CE, Reich $K$, et al. Baseline nail disease in patients with moderate to severe psoriasis and response to treatment with infliximab during 1 year. J Am Acad Dermatol 2008;58:224-31.

8 Bywaters E. Pathology of spondyloarthropathies. In: Calin A, ed. Spondyloarthropathies. London: Grune and Stratton; 1984:43-68.

9 McGonagle D, Stockwin L, Isaacs J, et al. An enthesitis based model for the pathogenesis of spondyloarthropathy. additive effects of microbial adjuvant and biomechanical factors at disease sites. J Rheumatol 2001;28:2155-9.

10 Benjamin M, McGonagle D. The enthesis organ concept and its relevance to the spondyloarthropathies. Adv Exp Med Biol 2009;649:57-70.

11 Jacques $P$, Venken $K$, Van Beneden K, et al. Invariant natural killer $T$ cells are natural regulators of murine spondyloarthritis. Arthritis Rheum 2010;62:988-99.

12 Diarra D, Stolina M, Polzer K, et al. Dickkopf-1 is a master regulator of joint remodeling. Nat Med 2007;13:156-63.

13 Correa-Meyer E, Pesce L, Guerrero C, et al. Cyclic stretch activates ERK $1 / 2$ via $G$ proteins and EGFR in alveolar epithelial cells. Am J Physiol Lung Cell Mol Physiol 2002;282:L883-91.

14 Cornelissen J, Armstrong J, Holt CM. Mechanical stretch induces phosphorylation of p38-MAPK and apoptosis in human saphenous vein. Arterioscler Thromb Vasc Biol 2004:24:451-6.
15 Papakrivopoulou J, Lindahl GE, Bishop JE, et al. Differential roles of extracellular signal-regulated kinase $1 / 2$ and p38MAPK in mechanical loadinduced procollagen alpha1(I) gene expression in cardiac fibroblasts. Cardiovasc Res 2004;61: 736-44.

16 Lal H, Verma SK, Smith M, et al. Stretch-induced MAP kinase activation in cardiac myocytes: differential regulation through beta1-integrin and focal adhesion kinase. J Mol Cell Cardiol 2007;43:137-47.

17 Sherlock JP, Joyce-Shaikh B, Turner SP, et al. IL-23 induces spondyloarthropathy by acting on ROR-gammat+ CD3+CD4-CD8- entheseal resident T cells. Nat Med 2012;18:1069-76.

18 Thomas GP, Brown MA. Genomics of ankylosing spondylitis. Discov Med 2010;10:263-71.

19 Castillo-Gallego C, Aydin SZ, Emery P, et al. Brief report: magnetic resonance imaging assessment of axial psoriatic arthritis: extent of disease relates to HLA-B27. Arthritis Rheum 2013;65: 2274-8.

20 Maksymowych WP. Ankylosing spondylitis-at the interface of bone and cartilage. J Rheumatol 2000;27:2295-301.

21 Lories RJ, Derese I, Luyten FP. Modulation of bone morphogenetic protein signaling inhibits the onset and progression of ankylosing enthesitis. J Clin Invest 2005;115:1571-9.

22 Lience $E$, Ros C, Sellas A, et al. Rheumatoid arthritis and hemiplegia: remission of the arthritis in the paretic limbs and appearance of a subcutaneous nodule in the non paretic elbow. Med Clin 1993;101:518-9.

23 Smith RD. Effect of hemiparesis on rheumatoid arthritis. Arthritis Rheum 1979:22:1419-20.

24 Braun J, van den Berg R, Baraliakos X, et al. 2010 update of the ASAS/EULAR recommendations for the management of ankylosing spondylitis. Ann Rheum Dis 2011;70:896-904.

25 Ward MM, Reveille JD, Learch TJ, et al. Occupational physical activities and long-term functional and radiographic outcomes in patients with ankylosing spondylitis. Arthritis Rheum 2008;59: 822-32.

26 Armaka M, Apostolaki M, Jacques $\mathrm{P}$, et al. Mesenchymal cell targeting by TNF as a common pathogenic principle in chronic inflammatory joint and intestinal diseases. J Exp Med 2008;205: 331-7. 